\title{
UNA NECESIDAD IMPERIOSA EN LA EDUCACIÓN GENERAL: EL DESARROLLO DE LA ACTIVIDAD COGNOSCITIVA PRODUCTIVA
}

\author{
Jorge Lázaro Hernández. Mujica \\ jorgelhm@ucpejv.edu.cu \\ UNIVERSIDAD DE CIENCIAS PEDAGÓGICAS \\ "ENRIQUE JOSÉ VARONA" \\ María Isabel Delgado Ortiz \\ mariaido@ucpejv.edu.cu \\ UNIVERSIDAD DE CIENCIAS PEDAGÓGICAS \\ "ENRIQUE JOSÉ VARONA"
}

Recibido: 4 de julio de 2018 Aceptado: 20 de noviembre de 2018

\section{Resumen}

Con el advenimiento del siglo XXI, se hace necesaria una educación para el futuro, en que se prepare a las nuevas generaciones para el cambio, para la variación de paradigmas científicos y tecnológicos, y no para la estabilidad, para lo rutinario, como ocurre actualmente en muchos centros docentes. ¿Qué significado tiene esta nueva exigencia? De esto se desprende la necesidad imperiosa de una activación productiva de la enseñanza, la cual ha sido una preocupación desde la Antigüedad. En Cuba, hay referencia de eminentes pedagogos; por ejemplo, José J. Martí Pérez (1853-1895), nuestro Maestro de todos los Maestros, insistió en esto, siempre con su proyección de futuro, desde el siglo XIX: "Cada hombre trae en sí el deber de añadir, de domar, de revelar. Son culpables las vidas empleadas en la repetición cómoda de las verdades descubiertas". “...el profesor no ha de ser un molde donde los alumnos echan la inteligencia y el carácter, para salir con sus lobanillos y jorobas...” ¿Por qué tanta insistencia en desarrollar la producción cognoscitiva? ¿Qué hacer en nuestras clases para contribuir a su logro? En el presente trabajo se propone fundamentar la necesidad del desarrollo de la actividad cognoscitiva productiva en la educación general.

Palabras clave: Actividad cognoscitiva productiva; enseñanza problémica; aprendizaje reflexivo.

\begin{abstract}
With the coming of the XXI century, it becomes necessary an education for the future in that gets ready to the new generations for the change, for the variation of scientific and technological paradigms, and it doesn't stop the stability, for the routine thing, like it happens at the moment in many educational centers. How meaning has this new demand? Of this he comes off the imperious necessity of a productive activation of the teaching,
\end{abstract}


which has been a concern from the Antiquity. In Cuba, there is eminent educators' reference; for example, José J. Martí Pérez (1853-1895), our Teacher of all the Teachers, insisted in this, always with his future projection, from the XIX century: "Each man brings in yes the duty of adding, of taming, of revealing. Are the lives guilty used in the comfortable repetition of the discovered truths". "...el professor a mould must be where the students toss the intelligence and the character, to go out with their cysts and humps..." why so much insistence in developing the cognitive production? What to make in our classes to contribute to their achievement? Presently work intends to base the necessity of the development of the productive cognitive activity in the general education.

Keywords: Productive cognitive activity; teaching problémica (problemic); reflexive learning. 


\section{1.- INTRODUCCIÓN}

“...la conjetura es uno de los medios del conocimiento (...) tengo el derecho de buscar la razón de lo vago por un camino racional. No fijaré lo que no sepa, pero investigaré lo que no sé". J. J. Martí Pérez (1853-1895)

En el curso del desarrollo histórico, el hombre fue capaz de desarrollar un nuevo tipo de actividad más allá de la simple actividad práctica: la cognoscitiva, cuyo objetivo esencial es el conocimiento de las propiedades y las relaciones de los hechos y los fenómenos del mundo circundante.

Generalmente, la actividad cognoscitiva se incluye dentro del desarrollo de los procesos intelectuales y, si bien es cierto que en esta se desarrolla el pensamiento, su percepción del mundo que lo rodea, su memoria consciente, la dirección voluntaria de su atención, también está presente la necesidad de adquisición del conocimiento, el deseo de saber y de conocer la forma de solucionar problemas.

En la medida en que los estudiantes descubren esa riqueza de interrelaciones entre los hechos y los fenómenos, y los domina creadoramente, surge en ellos la necesidad del saber. Penetrando en la esencia de los fenómenos del mundo, se forma un pensamiento dialéctico e independiente. Cuando aprenden a controlar su propia actividad, a dirigir sus esfuerzos, a perseverar en sus propósitos hasta vencer las dificultades, se desarrollan su control, su constancia y otros rasgos volitivos de su carácter.

En la enseñanza, en la escuela, los estudiantes, bajo la orientación del profesor, realizan una actividad cognoscitiva para asimilar los conocimientos, para lograr la formación de hábitos y habilidades, y adquirir procedimientos que les permitan plantearse posteriormente, por sí mismos, tareas de carácter cognoscitivo.

En la actividad docente, la asimilación de conocimientos científicos y la formación de habilidades correspondientes constituyen el objetivo y el resultado esencial de la propia actividad. En ella, el objetivo y el resultado son los cambios que se producen en los propios estudiantes que realizan la actividad y están determinados por la propia actividad de ellos, a diferencia de otros tipos de tareas, en que los cambios fundamentales se originan en el objeto sobre el cual se actúa.

La actividad cognoscitiva productiva, correctamente organizada y dirigida, propicia la iniciativa y la independencia de los estudiantes, y favorece condiciones favorables para la actividad creadora y el desarrollo de sus capacidades.

El objetivo del presente trabajo es fundamentar la necesidad del desarrollo de la actividad cognoscitiva productiva en la educación general. 


\section{2.- DESARROLLO}

La base de todo conocimiento humano es la actividad, que deviene de la relación sujetoobjeto y está determinada por leyes objetivas. Toda actividad está adecuada a fines, se dirige a un objeto y cumple determinadas funciones. V. González Maura y otros afirman que "...llamamos actividad a aquellos procesos mediante los cuales el individuo, respondiendo a sus necesidades, se relaciona con la realidad, adoptando determinada actitud hacia la misma. La actividad no es una reacción ni un conjunto de reacciones. En forma de actividad ocurre la relación sujeto-objeto, gracias a la cual se origina el reflejo psíquico que media esta interacción. Esto posibilita que pueda formarse en el individuo la imagen o representación ideal y subjetiva del objeto, y a su vez, pueda producirse la objetivación de la regulación psíquica en un resultado de la actividad" (González, M. y otros, 2004: 91).

“...el mal maestro informa la verdad, y el bueno enseña cómo encontrarla".

F. G. A. Diesterweg (1790-1866)

La actividad puede expresarse de dos formas: práctica o espiritual. La actividad práctica es determinante respecto a la espiritual; mediante ella se operan transformaciones en la realidad material que inciden, de una forma específica, en el mundo espiritual del hombre. La actividad espiritual implica cambios en las formas de la conciencia social; se determina como actividad gnoseológica, actividad cognoscitiva y actividad valorativoaxiológica. En sus inicios, ambas actividades estaban directamente vinculadas y formaban una unidad indisoluble. Con el desarrollo de la sociedad, la producción de ideas se separó de la producción de objetos y se transformó en una actividad teórica independiente, que tiene su propio contenido y especificidad: el conocimiento de las propiedades y las relaciones de los hechos y los fenómenos del mundo circundante.

"Si das un pescado a un hombre, se alimentará una vez; si le enseñas a pescar, se alimentará toda la vida."

Refrán chino

La actividad cognoscitiva constituye una forma esencial de la actividad espiritual del hombre. Según V. González Maura y otros, “... la actividad cognoscitiva es el proceso de penetración gradual en la esencia de los objetos y fenómenos, es el movimiento de lo desconocido hacia el conocimiento más completo y exacto, el conocimiento de las particularidades generales y esenciales de los objetos y fenómenos y de los vínculos entre ellos”(González, M. y otros, 2004: 144).Según R. Pupo Pupo (1990), la actividad cognoscitiva es un tipo de actividad espiritual que depende de la práctica, y la refleja y reproduce como generalizaciones esenciales que se manifiestan en la interacción sujetoobjeto. 
Para otros autores, “...la actividad cognoscitiva constituye la acción o conjunto de acciones proyectadas con vistas a conocer un objeto o aspecto del medio: ese es su fin $\mathrm{u}$ objetivo previamente determinado" (López, M. y Pérez, C., 1986: 33).

La actividad cognoscitiva constituye un complejo sistema que está relacionado con todos los aspectos de la personalidad humana, por lo que incluye componentes estructurales intelectuales, motivacionales y volitivo-emocionales. Estos componentes se ponen más de relieve cuando se trata de una actividad cognoscitiva estructurada, organizada y, consecuentemente, dirigida a un fin determinado, como es el caso de la actividad docente.

"No se crea empero que nosotros tratemos de proscribir enteramente el cultivo de la memoria. Lo que queremos evitar es que se efectúe en menoscabo de las demás facultades intelectuales".

José de la Luz y Caballero (1800-1862)

La actividad docente es la actividad cognoscitiva de los estudiantes, dirigida por medio del proceso de enseñanza-aprendizaje en la escuela. Durante su desarrollo los alumnos, bajo la orientación del profesor, deben asimilar conocimientos, lograr la formación de habilidades y adquirir procedimientos que les permitan plantearse posteriormente, por sí mismos, tareas de carácter cognoscitivo. Por tanto, la actividad cognoscitiva de los estudiantes se manifiesta en el proceso de enseñanza-aprendizaje como condición principal para el desarrollo pleno de la personalidad.

La actividad cognoscitiva de los estudiantes tiene ciertas particularidades que la diferencian de otros tipos de actividad, tales como: se realiza en la escuela, tiene un contenido previamente determinado y la asimilación de los conocimientos es un resultado de la actividad directa. En ella, se distinguen tres etapas:

- Planteamiento del problema (comprende fundamentalmente aspectos motivacionales).

- Ejecución (implica el dominio de las formas de trabajo docente).

- Regulación del proceso (análisis de los resultados).

En la actividad docente, la asimilación de conocimientos científicos y la formación de habilidades correspondientes aparecen como objetivo fundamental y resultado esencial de la propia actividad.

La actividad cognoscitiva puede ser de dos tipos (o grados, según López, M. y Pérez, C., 1986: 45): reproductiva o productiva (Figura 1). 


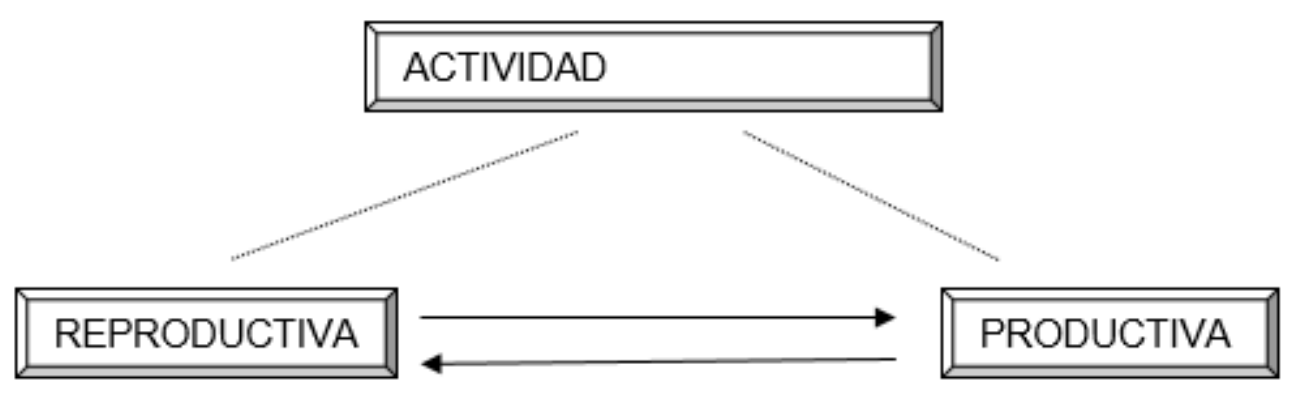

Figura 1. Tipos de actividad cognoscitiva

La actividad cognoscitiva reproductiva es valiosa y prepara las condiciones para ir transitando hacia la producción cognoscitiva. Por su parte, la actividad cognoscitiva productiva, en íntima relación con la actividad cognoscitiva reproductiva, constituye el tipo de actividad cognoscitiva caracterizada por la capacidad del sujeto de asimilar, transformar y aplicar los conocimientos adquiridos a nuevas situaciones de manera independiente y original, teniendo en cuenta sus vivencias y las características de su personalidad; por eso, hay autores que denominan a este tipo o grado de actividad cognoscitiva, como creadora.

"No hay cosa más horrenda que escuchar a todos los escolares respondiendo de la misma manera a las mismas preguntas."

Tomando como apoyatura didáctica lo anteriormente expresado, y concibiendo a la actividad docente como aquella actividad de la personalidad, desarrollada por medio del proceso de enseñanza-aprendizaje en la escuela, dirigida al proceso de obtención de los conocimientos y su aplicación creadora en la práctica social, la actividad docente puede organizarse teniendo en cuenta:

- Tarea docente. Se caracteriza por el planteamiento de una tarea, que lleva a los estudiantes a comprender que existe algo que no saben, algo para lo cual no tienen respuesta. Esta etapa tiene carácter motivacional. El profesor dirige la tarea docente y enfrenta a los estudiantes ante situaciones que hacen necesaria la búsqueda de un procedimiento general y de conocimientos específicos para la solución de diversos problemas, en correspondencia con el contenido. Se debe hacer énfasis en la formación de las acciones docentes de valoración. Se comprende la regulación del proceso y el análisis de los resultados, se forman las acciones de control y autocontrol, de valoración y autovaloración del proceso del trabajo docente y de sus resultados. En este proceso, los estudiantes aprecian sus insuficiencias, trabajan para su eliminación y comprenden la necesidad de acercar sus resultados al nivel establecido. También se debe tener en cuenta la importancia de la formación de conceptos. Los conceptos constituyen la forma 
fundamental con que opera el pensamiento. Relacionando los conceptos, se llega a formas más complejas del pensamiento, como son los juicios y los razonamientos. El concepto expresa el conocimiento de lo general y esencial en los objetos, los hechos y los fenómenos de la realidad. Cada ciencia tiene en su base un sistema de conceptos. Los estudiantes deben apropiarse de este sistema mediante el estudio de las diferentes asignaturas y, para lograr una correcta asimilación de los conceptos, resulta indispensable organizar y dirigir adecuadamente la actividad docente. En este contexto, se debe tener claridad en los tipos de conceptos: empíricos o especulativos y teóricos o científicos. El concepto empírico es resultado de este tipo de pensamiento y a él se llega como consecuencia de una generalización de las propiedades externas de los objetos y los fenómenos, obtenido fundamentalmente por comparación de cualidades comunes que se observan en un grupo determinado de hechos o fenómenos. El concepto teórico tiene en cuenta el sistema de relaciones internas que constituyen la esencia del objeto o fenómeno.

- Trabajo independiente. Una acertada dirección de la actividad cognoscitiva productiva implica la formación y el desarrollo, en los estudiantes, de habilidades para el trabajo independiente, lo que influye tanto en la adquisición de sólidos conocimientos como en la creación de las condiciones necesarias para que los estudiantes alcancen la independencia cognoscitiva. La escuela de hoy debe garantizar que los estudiantes adquieran los medios para lograr la asimilación de los conocimientos que se acumulan como consecuencia del vertiginoso desarrollo de la ciencia y la técnica, proporcionar los procedimientos para aprender; hacer vivir en los estudiantes la necesidad de saber, de actualizar su información, propiciar las posibilidades de asimilar los nuevos conocimientos y usarlos creadoramente (Pidkasisti, P. I., 1986).

- Enseñanza problémica (Figura 2). Para muchos autores, constituye un tipo de enseñanza basada en contradicciones dialécticas, que vinculan a los estudiantes con la investigación. Constituye una vía importante para potenciar la actividad cognoscitiva productiva, ya que propicia el enfrentamiento de los estudiantes a contradicciones que deben solucionar con su activa participación y de forma independiente, a fin de lograr un aprendizaje productivo; se relaciona con el proceso de búsqueda, de solución de problemas y de actividad creadora (Hernández, J.L., 1997: 7). El conocimiento de este tipo de enseñanza (sistema didáctico, para otros autores), así como su utilización en las clases, es aún muy pobre; la mayoría de los profesores carecen de un dominio adecuado de sus fundamentos teóricos y no la aplican consecuentemente, aun cuando creen hacerlo, confundiéndola con gran frecuencia con la llamada "enseñanza basada en problemas"; o sea, que en ocasiones se da incorrectamente el calificativo "problémico(a)". 


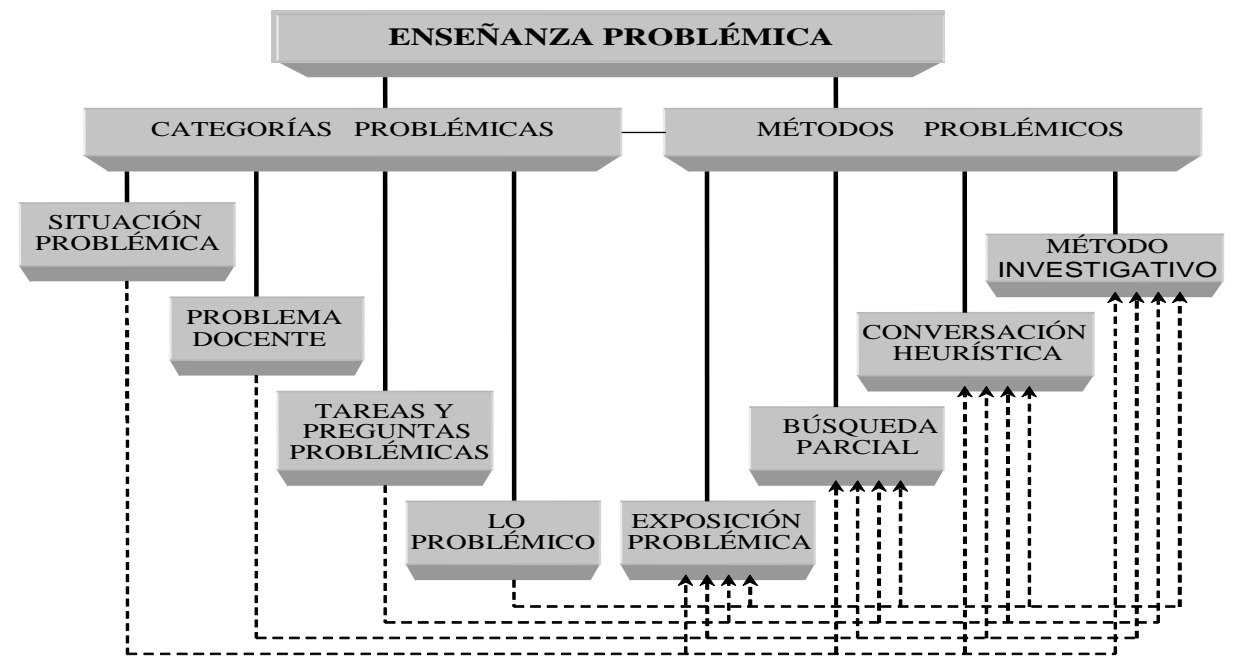

Figura 2. Relación entre las categorías y los métodos de la enseñanza problémica. Tomado de Hernández, J. L., 1997.

- Enfoque investigativo (Figura 3). Uno de los problemas más complejos que, en la actualidad, enfrentan las universidades es lograr, en los futuros profesionales de la educación, una actividad investigativa cotidiana, que les permita una actividad profesional reflexiva, crítica y transformadora de la realidad de la escuela. Hay que enseñar a los estudiantes a solucionar problemas educativos mediante la investigación en las escuelas secundarias básicas y los institutos preuniversitarios. Se hace necesario que desarrollen estrategias que favorezcan la búsqueda del contenido y que, al mismo tiempo, constituyan un modelo de actuación profesional pedagógica para su trabajo en la escuela. De esta manera, los estudiantes consideran el proceso de enseñanza-aprendizaje en su acción transformadora de la realidad, vinculando permanentemente teoría y práctica por medio de la investigación (Delgado, M.I., 2004).

- Aprendizaje reflexivo. Mediante este tipo de aprendizaje, los estudiantes transitan de un estado inicial a otro cualitativamente superior, como resultado de la actividad reflexiva y, por tanto, de la regulación de su propio proceso de aprendizaje, a partir de controlar y valorar, tanto la ejecución de sus acciones, como de los resultados que obtienen en el proceso de planteamiento y solución de problemas. Una de sus características esenciales es que los estudiantes se enfrenten a evidencias negativas que los induzcan a dudar de lo que aceptaban hasta ese momento (Velázquez, E., Ulloa, L. y Hernández, J.L., 2011: 45).

Tomando las anteriores variantes didácticas para desarrollar la actividad cognoscitiva productiva de las generaciones del siglo XXI, los autores del presente trabajo convocan a todos los profesores a que dirijamos el proceso de enseñanza-aprendizaje de 
nuestras respectivas asignaturas a preparar a nuestros alumnos con una cultura científica no solo para la solución de problemas de la vida cotidiana (es decir, percibir dificultades, captar situaciones malas, y buscarles solución, manteniéndolo ya alcanzado antes), cuestión muy necesaria, obviamente, sino es imperioso, además, que perciban las situaciones como desafíos, como oportunidades de crecimiento, es decir, planteamos un enfoque en que predomine un extremo interés por metas de mayor desempeño, en que se sobrepase el nivel antes alcanzado (Chibás, F., 2017: 7-10).

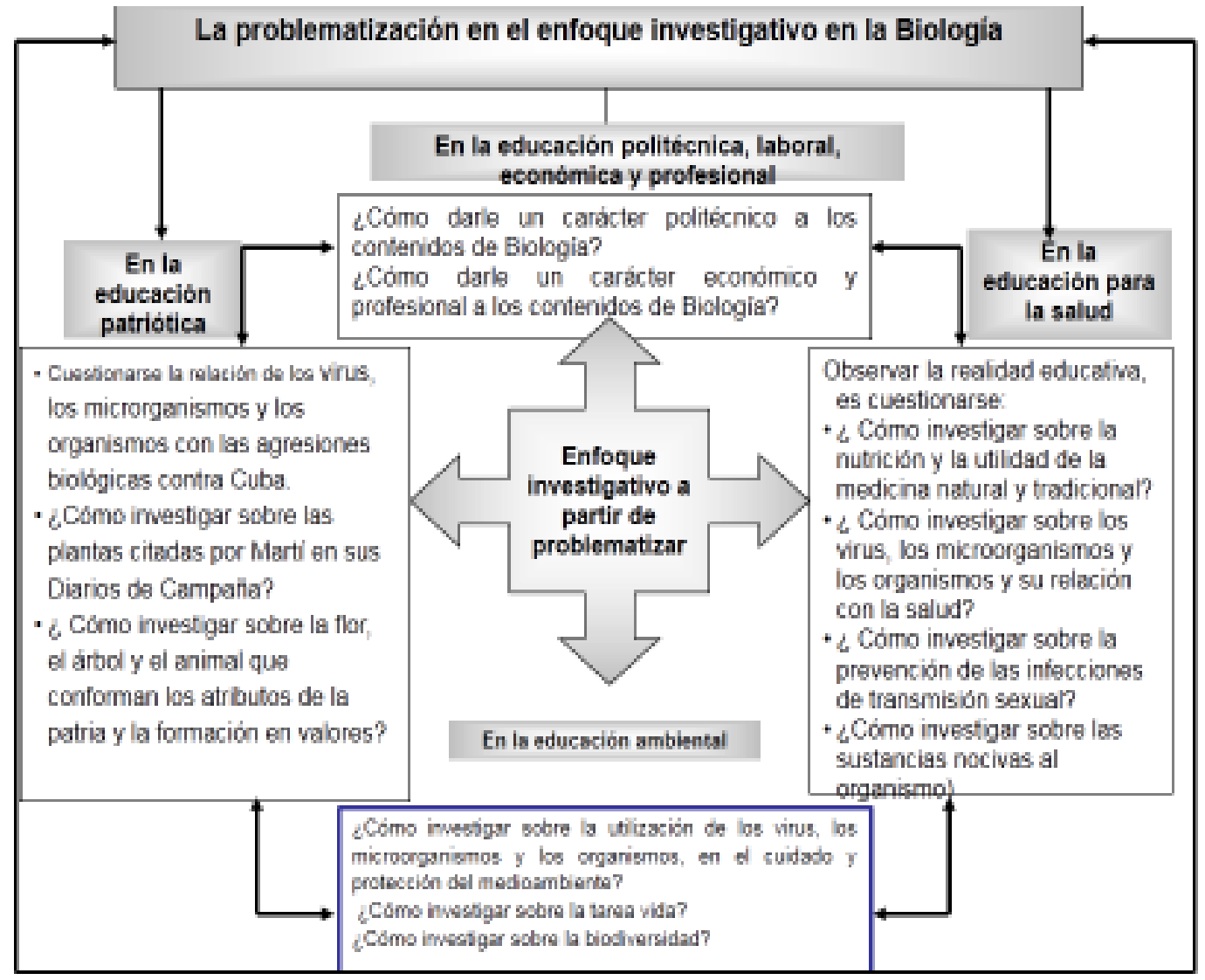

Figura 3. El enfoque investigativo en la enseñanza de las disciplinas biológicas. Tomado de Delgado, M.I., 2004

"Todos nosotros fuimos educados, enseñados con métodos pedagógicos antediluvianos. ¿En qué consiste el fallo principal de esos métodos? Que no se desarrollaba en el estudiante, en el joven, la iniciativa, que no se desarrollaba el instinto de observarlo todo, de indagarlo todo, de preguntarse acerca de todo, de analizar, de investigar."

Fidel Castro Ruz, 1974 


\section{3.- CONCLUSIONES}

- La educación actualmente tiene que preparar para la producción cognoscitiva o no es educación del siglo XXI; que debe cumplir su función formativa (educativa, desarrolladora e instructiva), a tono con la necesidad de una capacitación para el futuro, que se apreste al cambio, para la variación de paradigmas científicos y tecnológicos.

- La sociedad cubana actual demanda de una educación que potencie la actividad cognoscitiva productiva de los estudiantes y la actividad docente puede organizarse de manera que se pongan en función todos los recursos necesarios, tanto cognoscitivos como motivacionales y afectivos; para propiciar participación activa, independiente, a fin de lograr un aprendizaje productivo, reflexivo y creador.

\section{BIBLIOGRAFÍA}

ÁLVAREZ, C. M. (2011). Didáctica general: la escuela en la vida. Cochabamba: Grupo Editorial Kipus.

CHIBÁS, F. (2017). Creatividad, innovación y emprendedorismo. La Habana: Editorial Pueblo y Educación.

MINED-ICCP, Cuba (1984). Pedagogía. La Habana: Editorial Pueblo y Educación.

DANILOV, N. A. y SKATKIN, M. N. (1985). Didáctica de la escuela media. La Habana: Editorial Pueblo y Educación.

DELGADO, M. I. (2004). Estrategia didáctica para el establecimiento del enfoque investigativo integrador en la disciplina Microbiología de los institutos superiores pedagógicos. Tesis presentada en opción al Grado Científico de Doctor en Ciencias Pedagógicas. La Habana.

FERNÁNDEZ, A. y HERNÁNDEZ, J. L. (1989, octubre-diciembre). La aplicación de la enseñanza problémica en la Biología. En Revista Educación 75. La Habana, 89-96.

GONZÁLEZ, V. y otros (1995). Psicología para educadores. La Habana: Editorial Pueblo y Educación.

GUANCHE, A. S. (1997, enero-junio). Éxito de la enseñanza problémica de las Ciencias Naturales de la escuela primaria. Revista Varona 24. La Habana, 45-55.

(1999). Enseñanza problémica en las clases de Ciencias Naturales. Colección Promet. La Habana: Editorial Academia.

HERNÁNDEZ, J. L. (1997, enero-junio). La enseñanza problémica y la creatividad: producir versus reproducir, Revista Varona 24. La Habana, 40-44. 
(2013, julio-diciembre). Experiencia de la actividad creadora: vías para el tratamiento de este contenido del proceso de enseñanza-aprendizaje, Revista Varona 57. La Habana, 41-50.

Habana: Editorial Academia.

(1997). ¿Una ciencia para explicar Biología? Colección Promet. La

LABARRERE, A. F. (1996). Pensamiento: análisis y autorregulación de la actividad cognoscitiva de los alumnos. La Habana: Editorial Pueblo y Educación.

LÓPEZ, M. y MIRANDA C. (1986). La dirección de la actividad cognoscitiva. La Habana: Editorial Pueblo y Educación.

MAJMUTOV, M. I. (1983). La enseñanza problémica. La Habana: Editorial Pueblo y Educación.

MARTÍ, J. J. (1975). Obras completas. La Habana: Editorial de Ciencias Sociales.

MARTÍNEZ, M. (1981, octubre-diciembre). La enseñanza problémica. Revista Educación 43. La Habana, 82-91.

(1987). La enseñanza problémica de la Filosofía Marxista Leninista. La Habana: Editorial de Ciencias Sociales.

(1998). Calidad educacional, actividad pedagógica y creatividad. La Habana: Editorial Academia.

PIDKASISTI, P. I. (1986). La actividad cognoscitiva independiente de los alumnos en la escuela. La Habana: Editorial Pueblo y Educación.

PUPO, R. (1990). La actividad como categoría filosófica. La Habana: Editorial de Ciencias Sociales.

RODRÍGUEZ, M. y BERMÚDEZ, R. (2006). Diagnóstico psicológico para la educación. La Habana: Editorial Pueblo y Educación.

ROSENTAL, M. e IUDIN, P. (1981). Diccionariofilosófico. La Habana: Editora Política.

SALCEDO, I. M. y otros (2002). Didáctica de la Biología. La Habana: Editorial Pueblo y Educación.

TORRES, P. (1996). Didácticas cubanas en la enseñanza de la Matemática. Colección Promet. La Habana: Editorial Academia.

VELÁZQUEZ, E.A., ULLOA, L.G. y HERNÁNDEZ, J.L. (2011). Hacia un aprendizaje reflexivo. Berlín: Editorial Académica. 